\title{
Color-Changing Markers - An Exiting Topic for Inquiry-Based Chemistry Education
}

\author{
M. Ducci ${ }^{*}$ \\ University of Education Karlsruhe, Department of Chemistry, Karlsruhe, Germany \\ *Corresponding author: ducci@ph-karlsruhe.de
}

\begin{abstract}
Chemistry and life are undisputedly connected and always interdependent. Hence, it must be an essential aim to underline the value of this correlation in the light of our basic knowledge of chemistry and our general education. Moreover, it is important to relate chemical ideas with observations of daily life and living causes. In order to meet the aims of motivating and modern chemistry lessons, the author has developed a unit based on experiments exploring so-called Magic Markers. Ordinary looking felt pens change their colors when they are painted over by Magic Pens. To research on the chemical principles underlying this effect, number of simple but astonishing experiments suitable for students has been developed.
\end{abstract}

Keywords: Magic Marker, Dyes, Chemistry in Everyday Life

Cite This Article: M. Ducci, "Color-Changing Markers - An Exiting Topic for Inquiry-Based Chemistry Education." World Journal of Chemical Education, vol. 6, no. 1 (2018): 24-28. doi: 10.12691/wjce-6-1-5.

\section{Introduction}

For several years felt pens have been commercially available in many countries, exhibiting a very special property (refer to Figure 1): new colours are obtained by painting them over using the accompanying "Magic Pen“. An example is shown in Figure 2.

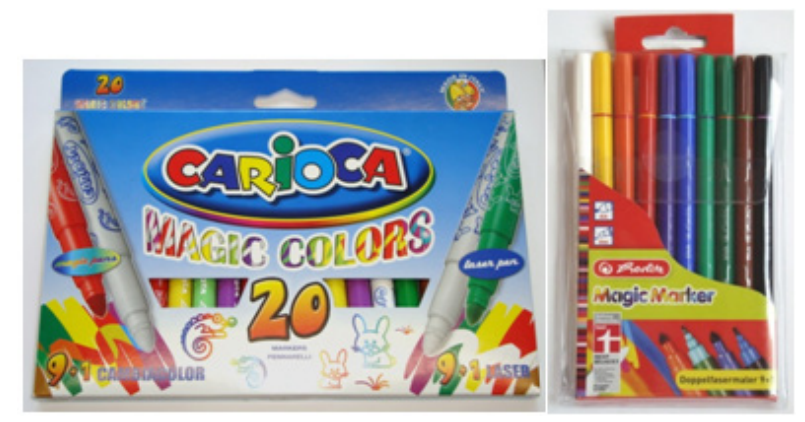

Figure 1. Magic Marker of Universal make (www.carioca.it; left) and of Herlitz make (www.herlitz.de; right)

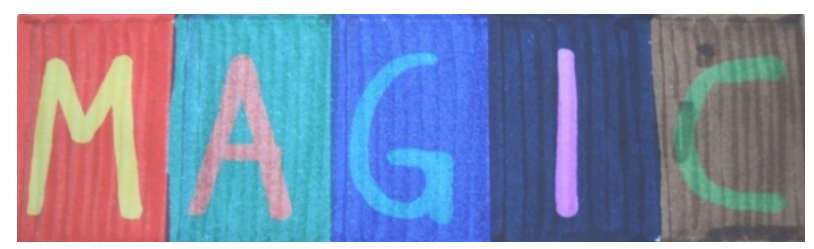

Figure 2. Colours of the Carioca Magic Markers to which a Magic Pen was applied

This motivating effect can be addressed and integrated in chemistry lessons at the upper secondary level to investigate the causes of the phenomenon. The author has developed a number of simple but amazing experiments to this end. Students can use these experiments in classes to investigate the underlying chemical processes.

This topic is excellently suited to address in an exemplary way both the basic principles of the colours of materials and the structure-property relations of the various dye classes as one of the essential basic concepts of chemistry. This topic and relating investigations enable the students to apply (and learn) important scientific methods common in chemistry as a natural science, e.g. photometric or chromatographic examinations. Moreover, the experiments have a meaningful context since the topic is related with everyday phenomena. Therefore, investigations into magic pens contribute to the development of the students' competence of thinking and action.

\section{The Concept}

The starting point of the investigations is the Magic Pen. The first task for the students is to check the effect of this pen on commercial fibre pens in their pencil cases. They find out that the Magic Pen can change the colour even some of these pens. An initially surprising result is obtained by painting over blue ink using the Magic Pen: the ink becomes colourless. At this stage many students start already to assume that the ingredients of the Magic Pen are similar to those of commercial ink erasers. This assumption is supported by the fact that the change of colours when painting over the Magic Marker colours with an ink eraser is identical to that obtained by using the Magic Pen for painting over (cf. Figure 3).

The next task for the students is to identify the ingredients of commercial ink erasers and find suitable analytical reactions for them, e.g. by performing an 
Internet search. Necessary information can be found on the websites of companies producing such ink erasers. The Pelikan homepage (www.pelikan.com), for instance, states that the erasing fluid of an ink eraser contains water, reducing agents and soda.

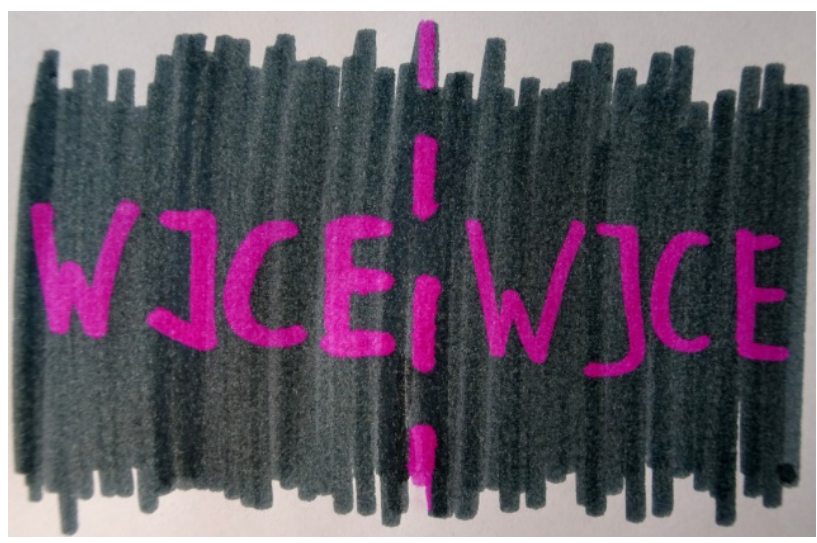

Figure 3. Colour of a Herlitz Magic Marker painted over with the Magic Pen (left) and a commercial ink eraser (right)

The students can draw the conclusion from this information that the fluid of the Magic Pen is alkaline due to the carbonate/hydrogen carbonate equilibrium if the same ingredients as in the ink eraser are used. This assumption is checked and verified in an experiment by wetting indicator paper with the Magic Pen fluid. The $\mathrm{pH}$ value of the Magic Pen fluid is about 11. The reducing agent can be detected, for instance, by using a potassium permanganate solution decolourising the reducing agent. The subsequent addition of barium chloride solution causing the formation of a white barium sulphate precipitate leads to the conclusion that sulphite ions were present in the initial solution (cf. Section 3, Test 1). This proves that the Magic Pen, similar to a commercial ink eraser, is filled with an alkaline, reducing solution (cf. Figure 4).

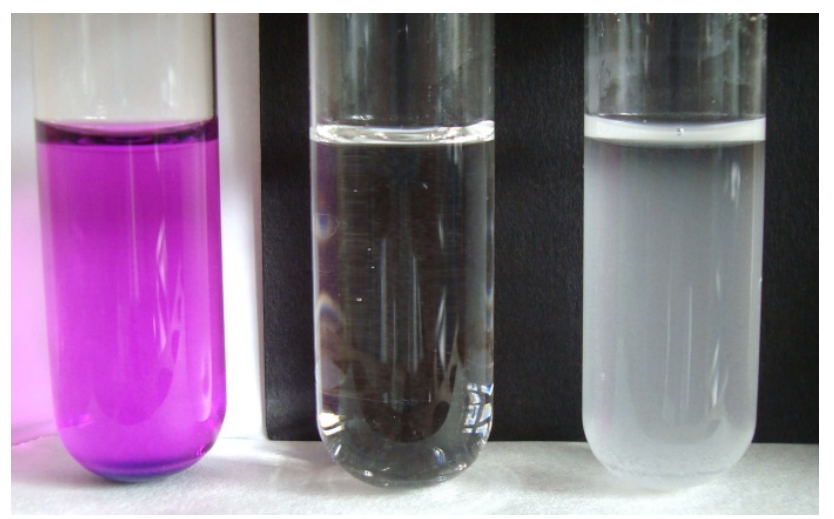

Figure 4. Potassium permanganate solution (left), solution after the addition of Magic Pen fluid (middle), white precipitate after the addition of barium chloride solution (right)

Then, the experimental investigations focus on the coloured Magic Markers. The students often assume that the dyes probably change their colour as a result of the high $\mathrm{pH}$ value of the Magic Pen fluid, i.e. that they are acid-base indicators. Hence, it must be assumed that the change of colours is caused by an alkaline solution even in the absence of sulphite ions and is reversible by acidification. In fact, this holds true for several pens, however other pens do not change colour in an alkaline environment at all or do not cause the change of colour desired by the manufacturer in such environment (cf. Section 3, Test 2 and Figure 5).

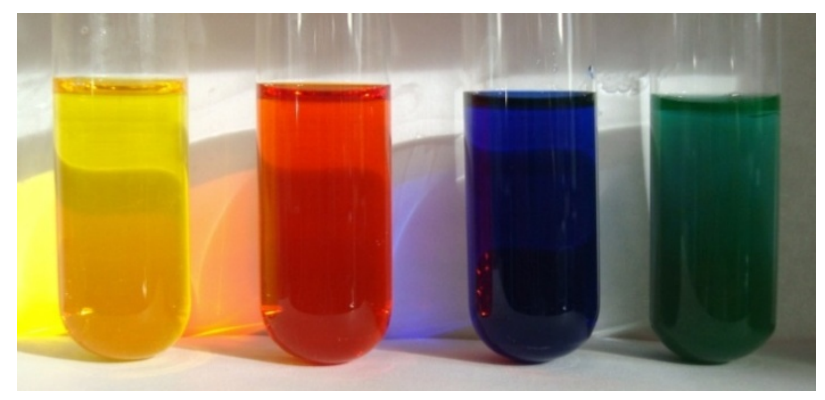

Figure 5. From left to right: Yellow dye solution, the same solution after the addition of caustic soda solution (this change of colour is desired by the manufacturer); blue dye solution, the same solution after the addition of caustic soda solution (however, painting over with the Magic Pen changes the colour from blue to yellow). Both changes of colour are reversible

Another proposal of students to find out the secret behind the change of colour is to perform a paper or thin layer chromatography. The chromatograms reveal that most of the pens contain dye mixtures consisting of two or more components. If half of the chromatogram is painted over by means of the Magic Pen or wetted with a sodium sulphite solution, the students come much closer to the secret behind the magic pens (cf. Figure 6).

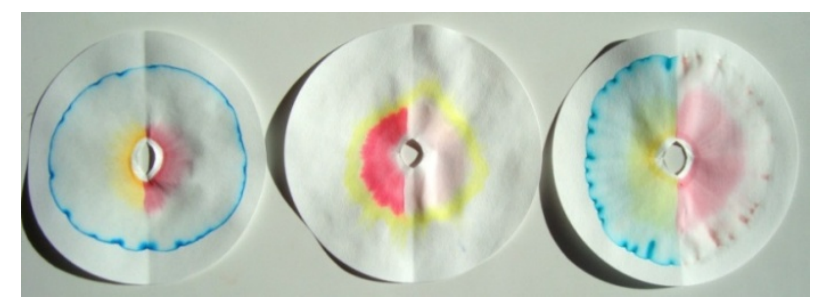

Figure 6. Paper chromatograms and wetting of the right half of each chromatogram using an alkaline sodium sulphite solution or Magic Pen fluid (from left to right: green pen with colour changing to purple (Herlitz), red pen with colour changing to yellow (Universal), green pen with colour changing to red (Universal))

Figure 6 shows that the colour changes are attributable to various effects. In several chromatograms the colour of individual components of the dye mixture change upon wetting with an alkaline sodium sulphite solution (e.g. Figure 6, left), in other chromatograms individual components are transferred into the colourless state (e.g. Figure 6, middle) or both an "eradication" of individual colours and a change of colour of one or several components occur at the same time (e.g. Figure 6, right).

Hence, the pens contain dyes, which are sensitive to high $\mathrm{pH}$ values and/or sulphite ions or are stable under these conditions ("stable" in the meaning of maintaining their original colour). The first group includes, for instance, many triphenylmethane dyes as well as numerous azo dyes. In contrast, for instance, xanthene dyes including also the rhodamines, are largely resistant to the above influences.

With this knowledge the dyes of several fibre pens are now identified. A possible approach is described here using the example of the green pen changing colour to purple (Herlitz). The paper chromatogram of this pen is 
depicted in Figure 6 (left). The chromatogram shows that this pen contains a yellow and a blue dye, the yellow dye changing colour to red upon wetting with the alkaline sodium sulphite solution. For a detailed analysis both dyes are separated from each other. This can be achieved, for instance, by means of column chromatography. In chemistry classes, however, it is more practicable to use paper chromatograms first to perform initial pre-tests.

For investigating the blue dye the paper chromatogram is cut into pieces and the blue coloured areas are placed into $5 \mathrm{~mL}$ of distilled water. The dye exhibits a very good solubility in water and changes its colour to green when being acidified. Very similar properties can be also observed, for instance, for the patent blue $\mathrm{V}$ and brilliant blue FCF dyes. The absorption spectra of these dyes in a neutral solution as well as the sample solution show identical absorption peaks at $630 \mathrm{~nm}$ for brilliant blue FCF and for the dye to be identified, whereas the absorption peak of patent blue $\mathrm{V}$ is at $639 \mathrm{~nm}$. Hence, brilliant blue FCF can be assumed to be the dye searched for (Figure 7).

Note: All absorption spectra were taken from diluted, translucent, aqueous dye solutions by using a Novaspec Plus spectrophotometer (path length of the cuvette: $1 \mathrm{~cm}$ ).<smiles>CCN(Cc1cccc(S(=O)(=O)O)c1)c1ccc(C(=C2C=CC(=[N+](CC)Cc3cccc(S(=O)(=O)[O-])c3)C=C2)c2ccccc2S(=O)(=O)[O-])cc1</smiles>

Figure 7. Structural formula of brilliant blue FCF

Applying drops of lye to the yellow areas of the paper chromatogram causes the yellow colour of the dye change to red. This change of colour is reversible by acidification. To roughly determine the colour transition range, various buffer solutions are applied. As shown in Figure 8 this indicator has a colour transition range between $\mathrm{pH} 7$ and $\mathrm{pH} 9$.

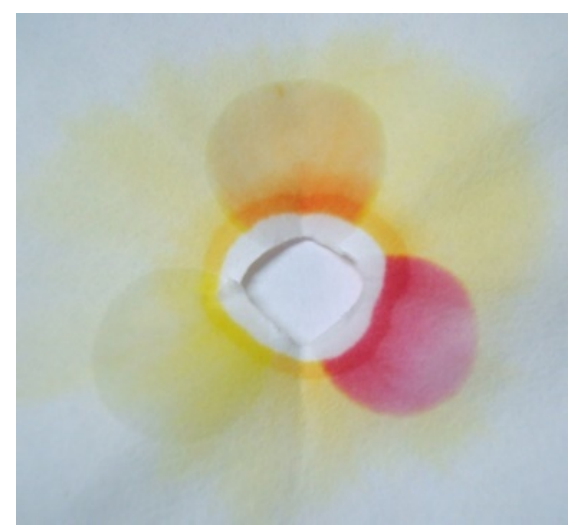

Figure 8. Detail from a paper chromatogram of the green Herlitz pen with drops of buffer solutions applied ( $\mathrm{pH} 7$ buffer solution left, $\mathrm{pH} 8$ buffer solution middle and $\mathrm{pH} 9$ buffer solution right)

Comparative tests with dyes exhibiting a similar chemical behaviour suggest that the dye may be brilliant yellow, i.e. an azo dye (Figure 9).

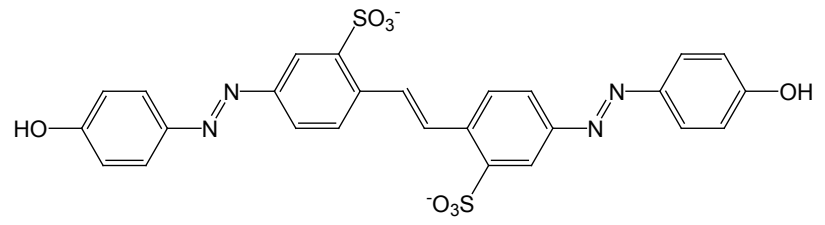

Figure 9. Structural formula of brilliant yellow

Further information on the yellow dye can be obtained from an absorption spectrum of the colour mixture from the green Herlitz pen. To this end the ink reservoir is removed and 1 drop of the coloured solution is added to $10 \mathrm{~mL}$ of a $\mathrm{pH} 5$ buffer solution. The absorption spectrum shows two peaks at $406 \mathrm{~nm}$ and $630 \mathrm{~nm}$ (Figure 10, blue line). The absorption spectrum of a mixture of brilliant yellow and brilliant blue FCF also shows two peaks of exactly the same wavelengths (Figure 10, red line).

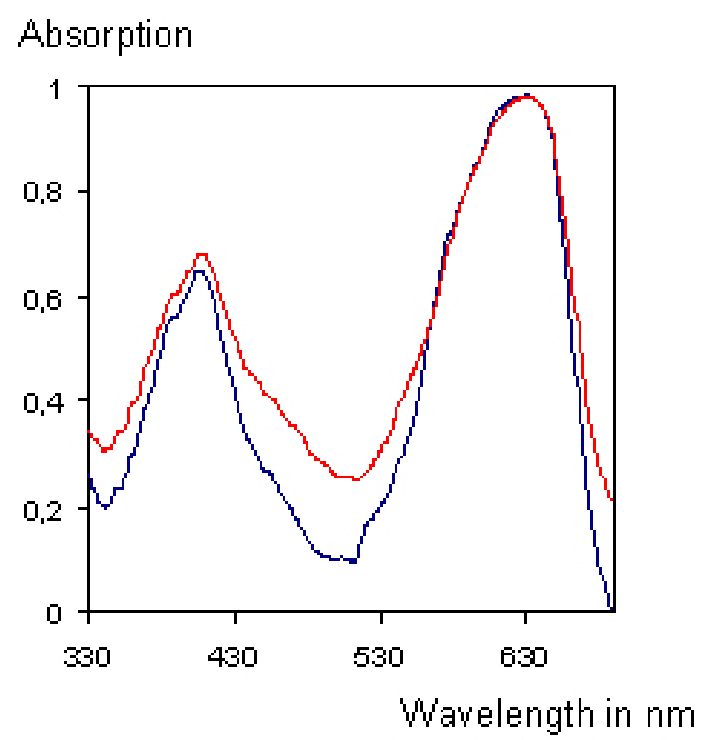

Figure 10. Absorption spectrum of the aqueous dye solutions (blue line: dyes from the Herlitz pen, red line: aqueous solution of brilliant yellow and brilliant blue FCF)

Here, another additional test should also be mentioned revealing that the yellow dye is an azo compound. Under certain conditions azo dyes can be broken down to aromatic amines by using strong reducing agents. And in fact, the colour of the green, aqueous solution of the dye mixture from the Herlitz pen changes its colour to blue following the addition of an acidic sodium dithionite solution (Figure 11, Test 3).

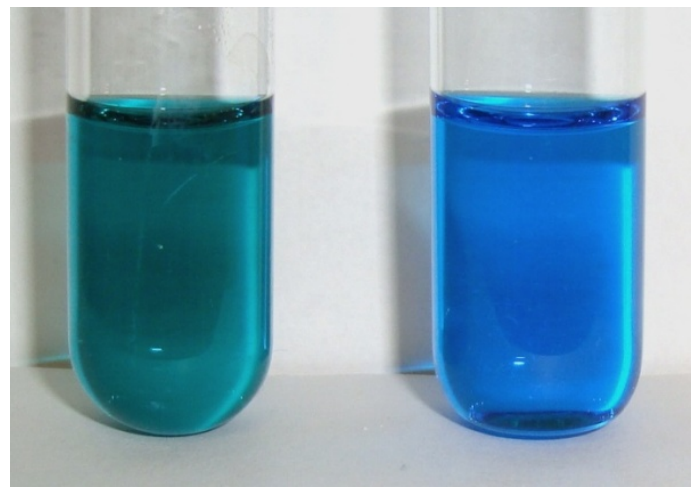

Figure 11. Aqueous dye solution prior (left) and after the addition of acidic sodium dithionite solution drops 
The absorption spectra of the solutions prior and after the reaction confirm that the peak at $406 \mathrm{~nm}$ has become clearly smaller. At the same time a new peak occurs at about $340 \mathrm{~nm}$, attributable to the reaction products of brilliant yellow (Figure 12).

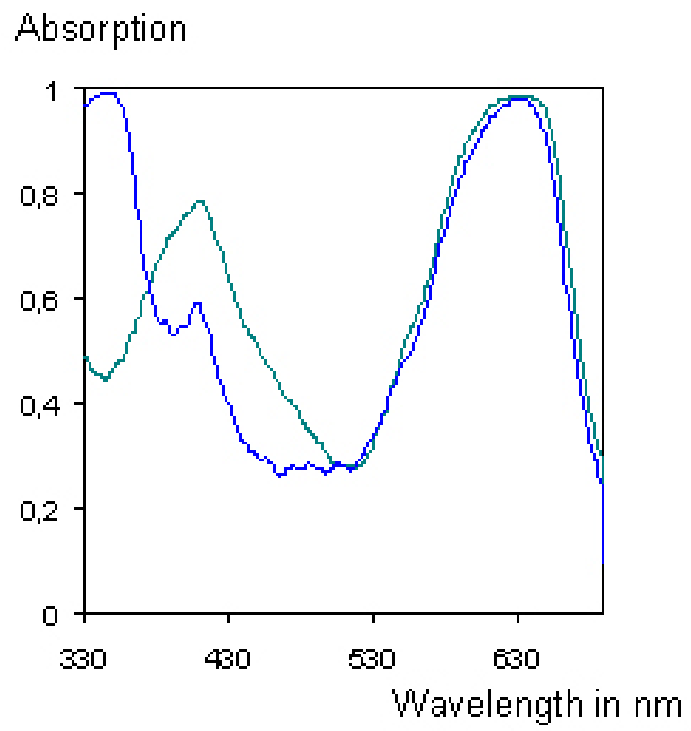

Figure 12. Absorption spectra prior to (green line) and after (blue line) the addition of sodium dithionite to the aqueous dye solution from the green Herlitz pen

Hence, the colour change of this pen is caused by deprotonation of the hydroxyl group of the azo dye transferring brilliant yellow to red, whereas brilliant blue FCF maintains its colour. Therefore, this change of colour may also be achieved by adding lye only.

As mentioned already, this does not hold true for all pens. An example is the blue pen of Carioca make exhibiting a change of colour to yellow fluorescent (Figure 13).

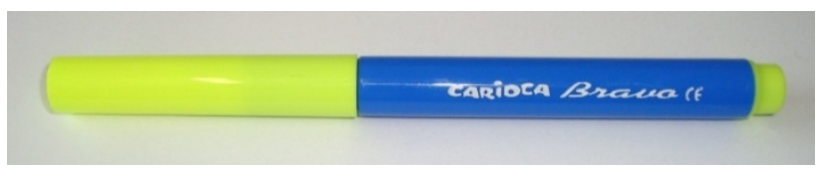

Figure 13. Magic Marker

The blue dye is methyl blue or water blue with a very similar structure; the yellow dye was identified to be pyranine, which is colourless in acids and exhibits a very intense yellow fluorescence in alkaline environments (Abb. 14) [1].

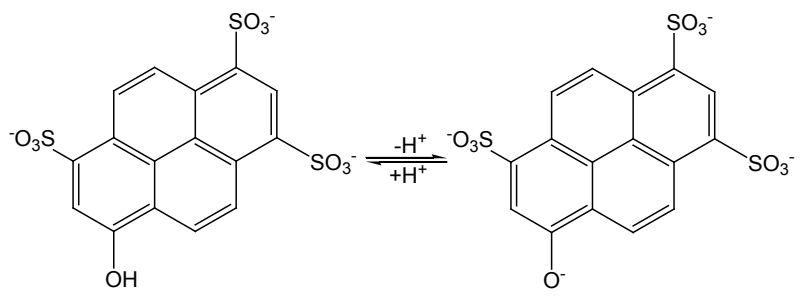

Figure 14. Pyranine

However, to obtain the desired change of colour the blue triphenlymethane dye needs to be transferred into the colourless state. This is achieved with sufficient speed only with the help of sulphite ions attaching to the central carbon atom. The large system of the delocalised $\pi$ electrons is destroyed since this carbon atom exhibits only four single bonds. The dye loses its ability to absorb visible light (Figure 15).

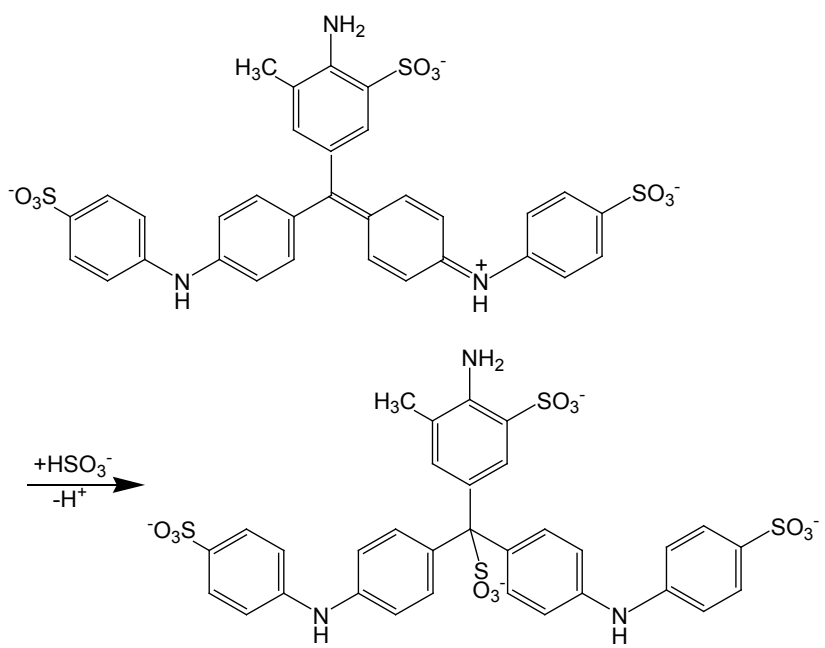

Figure 15. Discolouration of water blue by attaching a sulphite ion

\section{Experimental Investigations}

\section{Experiment 1: Examination of the Magic Marker fluid}

Equipment and chemicals: 3 test tubes, $10 \mathrm{~mL}$ measuring cylinder, $1 \mathrm{~mL}$ graduated pipette, Magic Pen (e.g. Carioca make), potassium permanganate, acetic acid, $\mathrm{c}\left(\mathrm{CH}_{3} \mathrm{COOH}\right)=2 \mathrm{~mol} / \mathrm{L}$, barium chloride, $\mathrm{w}\left(\mathrm{BaCl}_{2}\right)=5 \%$ [c: molar concentration; w: percentage by mass].

Test procedure: First, several crystals of potassium permanganate are dissolved in $10 \mathrm{~mL}$ of distilled water to produce a slightly coloured solution, which is acidified by adding $1 \mathrm{~mL}$ of acetic acid. The ink reservoir is removed from a Magic Pen and about 5 drops of the Magic Pen fluid are added to the potassium permanganate solution by pressing the ink reservoir. Subsequently, few drops of the barium chloride solution are added.

Observation: Following the addition of the Magic Pen fluid the solution is completely decolourised. The addition of barium chloride solution causes the formation of a white precipitate making the solution cloudy (Figure 4).

Interpretation: From the observations it can be concluded that the Magic Pen fluid contains sulphite ions. When reacting with permanganate ions they are oxidised to sulphate ions and are subsequently precipitated as barium sulphate:

$$
\begin{array}{ll}
\text { I. } & \mathrm{MnO}_{4}{ }^{-}+8 \mathrm{H}^{+}+5 \mathrm{e}^{-} \rightarrow \mathrm{Mn}^{2+}+4 \mathrm{H}_{2} \mathrm{O} \\
& \mathrm{SO}_{3}{ }^{2-}+\mathrm{H}_{2} \mathrm{O} \rightarrow \mathrm{SO}_{4}{ }^{2-}+2 \mathrm{H}^{+}+2 \mathrm{e}^{-} \\
\text {II. } & \mathrm{Ba}^{2+}+\mathrm{SO}_{4}{ }^{2-} \rightarrow \mathrm{BaSO}_{4} \downarrow
\end{array}
$$

\section{Experiment 2: Reactions of selected Magic Colour dyes} in an alkaline and acidic environment

Equipment and chemicals: 4 test tubes, dropping pipettes, 2 Magic Pens (yellow and blue pen with the colour changing to red respectively yellow, Carioca make), caustic soda solution, $\mathrm{c}(\mathrm{NaOH})=1 \mathrm{~mol} / \mathrm{L}$, hydrochloric acid, $\mathrm{c}(\mathrm{HCl})=1 \mathrm{~mol} / \mathrm{L}$ [c: molar concentration]. 
Test procedure: The selected pens are opened at the rear end to remove the refill. Then, two drops of the dye solution are filled into a test tube each by slightly pressing the ink reservoir and filled up with $10 \mathrm{~mL}$ of distilled water. Subsequently, 2 drops of the caustic soda solution first and thereafter 3 drops of the hydrochloric acid are added.

Observation and interpretation: The experiment shows that for the yellow pen the desired change of colour to red are caused by the caustic soda solution alone, the colour change being reversible by acidification (cf. Figure 5). The colour of the blue solution (reversibly) changes to green; however a yellow colour is produced when painting over with the Magic Marker. For the ingredients of these pens please refer to (Ducci, 2009).

\section{Experiment 3: Reductive cleavage of the yellow dye from the green Herlitz pen (colour change to purple)}

Equipment and chemicals: Herlitz pen (green pen changing colour to purple), pair of nippers, test tubes, dropping pipettes, sodium dithionite, hydrochloric acid, $\mathrm{c}(\mathrm{HCl})=0.01 \mathrm{~mol} / \mathrm{L}[\mathrm{c}$ : molar concentration $]$.

\section{Test procedure:}

The ink reservoir is removed from the Herlitz pen (it needs to be broken using, for instance, a pair of nippers) and one drop of the dye mixture is added to $5 \mathrm{~mL}$ of distilled water. The solution is divided into two parts, each of them being filled into one test tube.

Then, a solution of $5 \mathrm{~mL}$ of hydrochloric acid and 0.25 $\mathrm{g}$ of sodium dithionite is produced. 5 to 10 drops of this solution are added to the green dye solution in one of the test tubes.

Observation: Adding the drops of the acidic sodium dithionite solution causes the solution to become blue (cf. Figure 11).

Interpretation: The blue colouration is indicative of the fact that the yellow dye has been destroyed by reductive cleavage (formation of amines) (Figure 16). This can be confirmed by means of absorption spectra (cf. Figure 12).
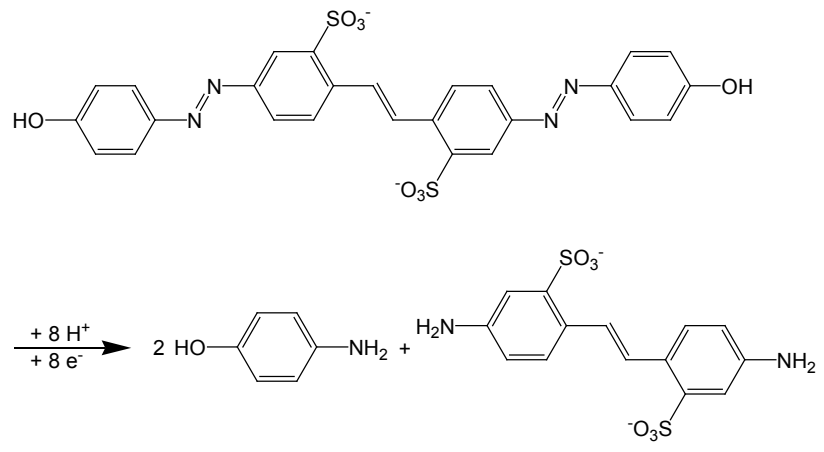

Figure 16. Reductive cleavage of brilliant yellow

\section{Results and Discussion}

This topic is excellently suited to address in an exemplary way both the basic principles of the colours of materials and the structure-property relations as one of the essential basic concepts of chemistry.

Student groups have always been highly motivated to lift the secret behind this exciting phenomenon of the colour-changing markers and are very proud having finally succeeded in understanding these initially unexplainable phenomena and attributing them to chemical principles.

With their newly acquired knowledge students were able to produce various Magic Markers and the Magic Pen themselves. This will be reported in a later publication.

\section{References}

[1] Herriger, C.; Klauck, M.; Ducci, M.: Color Changing Markers Ein spannendes Thema für den forschend-entwickelnden naturwissenschaftlichen Unterricht; PdN-Ch 58/7 (2009) 44-49 u. 51-52.

[2] Zajonc, S.; Gocke, S.; Ducci, M.: Magic Colours: Die Chemie der Zaubermaler - Ein Laborkurs im Rahmen des Projekts Make Science; CLB 62/11 (2011) 480-486.

[3] Ducci, M., Oetken, M.: Magische Stifte; Spektrum der Wissenschaft 11/16 (2016) 70-74. 